\title{
Consumer decisions with artificially intelligent voice assistants
}

\author{
Benedict G. C. Dellaert ${ }^{1,2}$ (D) Suzanne B. Shu ${ }^{3} \cdot$ Theo A. Arentze $^{4} \cdot$ Tom Baker $^{5}$. \\ Kristin Diehl ${ }^{6}$. Bas Donkers ${ }^{1} \cdot$ Nathanael J. Fast $^{6}$ - Gerald Häubl ${ }^{7}$. \\ Heidi Johnson ${ }^{8}$ • Uma R. Karmarkar ${ }^{9}$ - Harmen Oppewal ${ }^{2} \cdot$ Bernd H. Schmitt $^{10}$. \\ Juliana Schroeder ${ }^{11} \cdot$ Stephen A. Spiller ${ }^{12} \cdot$ Mary Steffel $^{13}$
}

Published online: 17 August 2020

(C) The Author(s) 2020

\begin{abstract}
Consumers are widely adopting Artificially Intelligent Voice Assistants (AIVAs). AIVAs now handle many different everyday tasks and are also increasingly assisting consumers with purchasing decisions, making AIVAs a rich topic for marketing researchers. We develop a series of propositions regarding how consumer decision-making processes may change when moved from traditional online purchase environments to AI-powered voicebased dialogs, in the hopes of encouraging further academic thinking and research in this rapidly developing, high impact area of consumer-firm interaction. We also provide suggestions for marketing managers and policymakers on points to pay attention to when they respond to the proposed effects of AIVAs on consumer decisions.
\end{abstract}

Keywords Artificial intelligence · Voice assistants · Consumer decision-making · Consumer dialogs $\cdot$ Digital marketing $\cdot$ Consumer models

Artificially Intelligent interactive Voice Assistants (AIVAs), also known as VoiceActivated Personal Assistants or Smart-Home Personal Assistants, have become widely adopted by consumers as aids in a variety of everyday tasks. AIVAs currently handle over one billion tasks per month, with the majority of uses being simple information requests ("Cortana, what is the weather today?") or household commands ("Ok Google, turn on the lights.").

AIVAs are also increasingly assisting consumers with purchasing decisions. Surveys indicate that voice shopping will jump from $\$ 2$ billion in 2018 to $\$ 40$ billion by $2022 .{ }^{1}$ Such surveys report that much of this shopping is simple re-ordering ("Alexa, add

\footnotetext{
${ }_{1}^{1}$ https://www.prnewswire.com/news-releases/voice-shopping-set-to-jump-to-40-billion-by-2022-rising-from-
} 2-billion-today-300605596.html

Benedict G. C. Dellaert

dellaert@ese.eur.nl

Extended author information available on the last page of the article 
paper towels to my shopping cart."), but AIVAs have the potential to be more interactive, include experiential service purchases (e.g., what restaurant to visit), and serve as partners in decision dialogs rather than mere order takers. It is this interactive verbal decision process, and what distinguishes it from traditional web-based online purchases, which we focus on in this paper.

While much is known about how consumers make decisions in digital environments characterized by screen-based interactions and online recommendation systems (e.g., Diehl et al. 2003; Häubl and Trifts 2000; Xiao and Benbasat 2007), far less is known about how consumer decisions are made in dynamic dialogs with an AIVA. Consumers' decision processes in verbal dialogs likely differ from those in visual environments and may be subject to new idiosyncratic decision biases (e.g., Munz and Morwitz 2019). We propose this has consequences for consumer adoption of AIVA technologies, consumer decision-making processes, and outcomes, and hence for how firms and policymakers can best respond to these effects of AIVAs on consumers.

Our first aim in this paper is to encourage further academic thinking and research by suggesting key aspects in which consumer decision-making may change in the presence of AIVAs compared with traditional online purchase environments. We define AIVAs as entirely voice-based interfaces that can actively guide consumer decisions on the basis of artificial intelligence. We propose that interacting with an AIVA, as compared with using traditional online purchase environments, presents a unique consumer experience, with implications for the decision to adopt AIVAs (Section 1) and the decision process itself (Section 2). We develop a set of behavioral propositions for future research to explore these implications. Our second aim is to provide suggestions for marketing managers and policymakers on points to pay attention to when they respond to the proposed effects of AIVAs on consumer decisions (Section 3).

\section{Consumer adoption of Al-powered voice assistants}

Choosing by holding a dialog with an AIVA may involve several trade-offs between autonomy and efficiency that are not inherent in choosing with a traditional online system. These potential trade-offs will affect adoption. During information search, people need to decide whether to maintain control over consideration set options or instead accept help in simplifying market offers. This trade-off may be greater when choosing with an AIVA because it involves sequential processing which takes longer and requires more working memory capacity than visually skimming descriptions of options presented simultaneously. ${ }^{2}$ Consequently, people will rely more on the AIVA's prioritization of alternatives and consider fewer options, thus trading sovereignty over their preferences for guidance in selecting the best option. This trade-off may be felt even more acutely because engaging in a decision dialog with an agent with a humanlike voice (e.g., natural pitch variance) may feel more akin to sharing choice responsibility with another person (Epley et al. 2013; Schroeder and Epley 2016;

\footnotetext{
${ }^{2}$ While we focus exclusively here on common voice-only assistants, the growing market prevalence of AIVAs with visual displays is evidence of certain limitations of the audio-only sequential process; it is an open question how decision processes change when both audio and visual feedback is available.
} 
Schroeder and Epley 2015), which may make consumers feel less personally responsible for the decision (Harvey and Fischer 1997; Steffel and Williams 2018; Steffel et al. 2016). When implementing decisions, people trade off oversight for help executing decisions efficiently. This tradeoff is amplified when choosing with an AIVA due to the ephemeral nature of verbal conversations compared with written interactions; the execution of the decision may be more difficult to track and monitor over time.

These trade-offs between autonomy and efficiency may affect whether people seek decision support from AIVAs and for what purpose. People may prefer to share decision autonomy with an AIVA when they wish to avoid the burden of responsibility associated with decisions, such as with difficult choices they worry they might regret (Steffel and Williams 2018) and choices for others for which they worry they might be blamed (Steffel et al. 2016). People may prefer not to rely on an AIVA when decision autonomy is tied to their sense of self-determination (Ryan and Deci 2000) and selfesteem (Usta and Haubl 2011). Relatedly, people may be more reluctant to adopt AIVAs for evaluating alternatives than for gathering information or executing a decision, as weighing options and making a decision is more likely to involve the self-concept. Perhaps, this is one reason why current AIVAs focus primarily on information search and task implementation.

\section{Proposition 1: Greater consumer willingness to trade off autonomy for efficiency will increase adoption of AIVAs, which involve a greater autonomy-efficiency tradeoff than traditional online systems.}

Also affecting adoption, consumers interacting with an AIVA will have stronger psychological reactions to the system's perceived human-like behaviors. On the one hand, interacting with a human-like AIVA may make consumers feel powerful (Fast and Schroeder 2020), leading to a preference for adopting AIVAs for personal use. On the other hand, AIVAs that are too human-like in appearance and/or communication could inadvertently trigger a feeling of discomfort among consumers (Mori et al. 2012; Wang et al. 2015). Kim et al. (2019) showed that when consumers perceive robots as warm in appearance or behavior, they initially judge them as positive, but as robots become increasingly warmer and more human-like, the uncomfortable feeling of uncanniness sets in and diminishes positive attitudes. While the aforementioned research suggests that being overly human-like may be detrimental for an AIVA, algorithms can still be implemented in ways that appeal to the consumer. In particular, we propose that for subjective (i.e., personalized and difficult to quantify) decisions, humanized algorithms will be preferred over seemingly mechanistic algorithms. For example, consumers prefer humans for subjective tasks but AI for objective tasks (Castelo et al. 2019a; Newman et al. 2020); whether an AIVA is adopted more when labeled as AI or human could depend on the task it is asked to perform (Castelo et al. 2019b).

Proposition 2:Greater consumer psychological comfort with AIVAs, which will depend on the alignment between the type of decision task and the human likeness of the algorithm, will increase their adoption.

More than other recommendation systems, AIVAs rely on consumers' capacity to mentally represent the decision alternatives to effectively engage in voice-based dialogs. Any situational factors that impair consumers' ability to mentally simulate choice 
options by lowering their attention or limiting their processing capacity should reduce consumers' willingness to use AIVAs. AIVAs also rely more heavily on consumers' prior knowledge than traditional systems. Greater prior knowledge allows consumers to better mentally simulate a product or its use (Alba and Hutchinson 1987). Further, a more detailed consumption vocabulary allows them to better voice their preferences (West et al. 1996). Hence consumers with greater prior knowledge may be more likely to adopt an AIVA. High-knowledge consumers may also be able to use AIVAs more efficiently by posing more specific queries and assessing more readily whether or not a given response fits their request. With regard to the properties of the decision alternatives, consumers may be less likely to use AIVAs for products, categories, and decisions that are more difficult to represent mentally.

Proposition 3: Greater consumer capacity to mentally represent the decision alternatives will increase adoption of AIVAs.

\section{Consumer decision-making with Al-powered voice assistants}

Interacting with AIVAs will also alter the decision process itself. Using an AIVA may create a unique experience that is distinct from other human-technology interactions (Lieberman and Schroeder 2020). When it comes to influence on choices, an AIVA's ability to signal warmth (e.g., cues like gender, affect, tone) and competence (e.g., immediate answers, a broad array of facts, real-time information) simultaneously enhances trust in the AIVA's motives as well as the quality of its recommendations. As a consequence, users may be more susceptible to AIVAs' influence over their choices. When people are aware that AIVAs are not fully human, they may be freed from the evaluation pressures they tend to experience with humans (Raveendhran and Fast 2019a; Raveendhran and Fast 2019b). Lucas et al. (2014) found that people tend to disclose more personal information to virtual humans, in the form of a computer avatar, than to actual humans. The awareness that AIVAs are not actual humans may lead people to use them for assistance with more personal issues and, simultaneously, may reduce embarrassment about their choices. In contrast, socially evaluative comments by the AIVA (e.g., "are you sure?") may lead to negative reactions and increase evaluation concerns.

Proposition 4: AIVAs increase consumer susceptibility to seller influence compared to traditional online purchase environments.

Consumers tend to focus on relatively narrow mental decision construals (Slovic 1972). While prior research is grounded in visual environments, auditory environments will likely exacerbate this tendency. As AIVAs limit contextual cues and the amount of information that can be processed simultaneously, fewer alternatives will be considered. This can have negative effects, such as excluding more-favored options (Nedungadi 1990), as well as positive effects, such as avoiding poor decisions when choosing among many mediocre options (Diehl 2005). In addition to considering fewer options, interacting with AIVAs may also focus consumers on a limited number of attributes because searching additional information may seem costly. Further, AIVAs 
may influence whether consumers process by alternative or by attribute (Payne et al. 1993). Given the linear format of AIVAs and the marketplace's tendency to provide information by alternative, consumers using AIVAs may be particularly likely to process information by alternative, which makes comparisons harder and highlights within-alternative trade-offs.

The tendency to consider fewer options and to process options by alternative suggests that consumers may be more likely to evaluate options separately rather than jointly (Munz and Morwitz 2019). Using AIVAs makes it harder for consumers to make large jumps to other parts of the decision space (i.e., to different alternatives, different attributes, or evoking different goals; Meißner et al. 2020). However, such jumps may improve decision outcomes in environments where orderings are not very good (Diehl 2005). Thus, AIVAs are likely to lead to local rather than global optimization (Häubl et al. 2010).

Proposition 5: AIVAs reduce the scope of options considered compared to traditional online purchase environments, leading to local rather than global optimization.

The local focus described in proposition 5 suggests that AIVA-supported decisions will be particularly sensitive to path dependence, especially the impact of different starting points and local decision environments. This may have several implications. As evaluations shift in accordance with initial steps in the decision process (Ge et al. 2015; Simon and Spiller 2016), this may influence subsequent information acquisition. Further, explicitly going back and negating earlier steps may seem aversive because it reduces feelings of progress, even if backtracking ultimately may be more efficient (Soman and Shi 2003). Collectively, these factors may reduce consumers' tendency to engage in exploratory behavior when using an AIVA. Exploration can lead to dead ends and recovering from such dead ends may be difficult in voice-guided decisions due to effortful backtracking. As consumers begin to anticipate such costs, exploration may decline. At the same time, by reducing exposure to other alternatives and features of other alternatives, AIVAs may lead to less buyers' remorse and less regret over foregone options (Griffin and Broniarczyk 2010; Shu 2008).

Proposition 6: AIVAs amplify path dependence in the consumer decision process compared to traditional online purchase environments, which can have both positive (less regret) and negative (less exploration) consequences.

\section{Implications for marketing managers and policymakers of consumer decisions with AIVAs}

Thus far, we have considered how using an AIVA to guide consumer purchase decisions can change these decisions and have offered testable propositions for these effects. We now turn to suggestions for points of attention for marketing managers and policymakers as a consequence of these proposed changes. Table 1 summarizes these suggestions. 


\subsection{Suggested attention points for marketing managers}

While marketing managers often used a formal model of consumers' minds to support decision-making in traditional online purchase environments, we suggest that the complexity of the AIVA interaction process requires more extensive formalization of individuals' mental models than in traditional online systems. We propose two dimensions by which this complexity is introduced. First, following proposition 2, voicebased assistance will likely benefit from models that allow for a more abstract representation of options when matching them to individuals' needs and wording in decision dialogs. While traditional recommendation algorithms typically represent options in terms of their (tangible) attributes, individuals in voice-based dialogs may use more abstract benefits to express their needs. Thus, models that connect attributes to abstract benefits are particularly relevant for AIVAs (Arentze et al. 2015). For example, if the AIVA is aware of a consumer's (abstract) desire to lose weight, it may highlight caloric attributes of food options. Similarly, AIVAs can benefit from an ability to relate to a person's emotional state and empathize with the user. Interactive systems that can recognize and express emotions as well as be able to recognize, interpret, and act upon social signals are likely to be more successful (Yalcin and DiPaola 2018). Thus, marketing managers can pay attention to AIVAs' ability to model the consumer's mind in terms of capturing the consumer's (a) needs-based representation of reality, (b) dynamic relationship with reality, and (c) emotional state.

Second, based on proposition 6 , we expect that due to the inherently sequential nature of voice-based interactions, models used by AIVAs will benefit from being able to encode dynamic aspects of a consumer's representation of a decision problem. The AIVA can track how the individual's current state relates to previous interactions. Individuals may forget certain information over time, and an AIVA may not assume that the individual still knows previously provided information. It is also beneficial to capture how the current state relates to the future. For example, individuals may have goals that they wish to achieve, and a voice-based assistant will need to be able to monitor progress toward these (future) goals when providing (current) advice.

Thus, for AIVAs to interact with users in a naturalistic, voice-driven manner, the dialog system will likely need to not only understand a diverse set of inputs but also to respond in a similar fashion, relying on the same language terminology as the user. Current human language systems can continue user-initiated dialogs while accounting for path dependence. To be more assistive, the conversation could also help the user achieve the purpose of the interaction. In other words, output from AIVAs may benefit from accurately translating formal models into the consumer's language.

Other recommendations for managers follow from propositions 1 and 3. In particular, we suggest that at each point in the dialog, a system can choose from a wide range of responses to help the user achieve his or her goals. A system should understand the user's mental model, the short-term goal for the current interaction, and long-term goals and general interests. This requires access to a variety of personal data and integration into a broader system of purchase and behavior records. Depending on the user's mental state, different continuances of the dialog will be more or less effective. A key component missing from most current AIVAs is the ability to give purpose to an extended dialog, such that the dialog moves in the desired direction. Importantly, such models need to choose the response that will most effectively move the dialog forward, such as asking for important information that 
Table 1 Consumer decisions with AIVAs: research propositions and suggested points of attention for marketing managers and policymakers

Research propositions

Suggested attention points

Adoption of AIVAs

1. Greater consumer willingness to trade off autonomy for efficiency increases adoption

- Provide advice that matches consumers' desire to progress on future goals (marketing managers)

- Promote personalized choice engines to help protect consumers in complex decision environments (policymakers)

2. Greater consumer comfort, which depends on the alignment between the decision task and the human likeness of the AIVA, increases adoption

- Provide abstract option representations when matching to individuals' needs and wording (marketing managers)

- Build systems that can recognize and express emotions (marketing managers)

- Be cautious of systems that use word choice (slang or jargon) and/or emotional signals to categorize consumers by social class or economic signifiers (policymakers)

3. Greater consumer capacity to mentally represent the decision alternatives increases adoption

- Develop models that include purpose in extended decision dialogs and move the decision process forward effectively, using "pull" and/or "push" dialog to support the consumer (marketing managers)

Decision-making with AIVAs

4. AIVAs increase consumer susceptibility to seller influence

- Develop mandatory disclosure requirements for AIVAs that include information that sellers do not have an incentive to produce or reveal (policymakers)

- Require that AIVAs function as the agent (with a fiduciary obligation) for the consumer they are assisting (policymakers)

5. AIVAs reduce the scope of options consumers consider, leading to local rather than global optimization

- Recognize that consumer risks posed are particularly acute in verbal decision environments because choice sets are more limited than for written information (policymakers)

- Monitor possible discrimination against vulnerable populations via omission, such as absent options or missing information (policymakers)

6. AIVAs amplify path dependence in the consumer

- Develop models that can encode dynamic aspects of decision process a consumer's representation of a decision problem during sequential processing of options (marketing managers)

is missing ("pull" dialog) or providing information to the user that $\mathrm{s} / \mathrm{he}$ is missing ("push" dialog). This approach not only applies to objective knowledge but also to subjective aspects of the dialog, where a message like "This looks good!" can provide reassurance to the user.

\subsection{Suggested attention points for policymakers}

The proposed effects of using AIVAs for consumer decisions also raise several policyrelated points of attention. With respect to consumer adoption of AIVAs, the White 
House Task Force on Smart Disclosure has noted several potential benefits of electronic information for consumers, including the opportunity for improved decisionmaking when using choice engines fueled by these data. This is especially relevant for decisions where consumers have a low need for autonomy, consistent with proposition 1. Personalized choice engines may be especially valuable for such low autonomy decisions, and their potential have been recognized by policymakers such as in the Dodd-Frank Wall Street Reform and Consumer Protection Act of 2010.

However, since consumers may be more susceptible to seller influence (proposition 4 ), active regulatory requirements may become more necessary. One way to mitigate the risks that AIVAs pose for consumers is for consumer-facing firms to adopt machine-readable disclosures. For example, financial services firms can be required by the Consumer Financial Protection Bureau (CFPB) to provide consumer information in a standardized, machine-readable form. Machine-readable disclosure information could manifest through an AIVA's tailored and personalized sorting of information to an individual consumer. Mandatory disclosure requirements include information that companies do not have an incentive to produce or reveal; similarly, companies may not have an incentive to provide this information in a form that AIVAs can use without regulation. However, requiring machine-readable information does not guarantee that AIVAs (or consumers) will use the information (Loewenstein et al. 2011). Disclosure requirements are only meaningful if the information is integrated with an AIVA's recommendation system.

In addition, the risks posed to consumers are particularly acute in verbal decision environments because the choice set is likely to be more limited than in decision environments where consumers view written information (i.e., proposition 5). Regulators could monitor whether AIVA companies voluntarily choose to incorporate disclosure information in their algorithms. Where gaps exist, regulators may need to consider extending the responsibility for providing disclosure information to the AIVA parent companies themselves.

Beyond regulation, proposition 4 further suggests a role for contract law in governing AIVAs and other digital assistants. Contract law offers no significant promise of protecting consumers from assistants that are biased against them. Creating an enforceable contract requires only that an assistant accurately discloses the nature of the service that it is providing, and on whose behalf, with no limit on the length of the disclosure (which can create the familiar "click through" disclosure that almost no one reads). Moreover, contract law offers only weak and difficult to access remedies for even highly abusive practices that are consistent with the terms of the disclosed contract. Consumer protection law offers somewhat more protective standards, prohibiting "unfair and deceptive practices," and potentially more powerful statutory remedies, but practical enforcement of those standards typically requires a government consumer protection agency, such as the Federal Trade Commission or a state attorney general, to take action (Sawchak and Shelton 2017).

One promising approach to AIVAs could be to require that they function as the agent (with a fiduciary obligation) for the consumer they are assisting. As a fiduciary, an AIVA would have a legal obligation to place the interests of the consumer first, ahead of the interests of the company that provided the AIVA or the company's contracting partners. For example, a fiduciary could not rank an insurance product higher based on the benefit that the insurance firm provides to the AIVA company. 
Because AIVAs can be manufactured to keep a record of the programs according to which they operate and the actions they take, those operations and actions can be audited, ideally on an automated basis, to verify that the assistant is complying with the standard (Baker and Dellaert 2018). When the AIVA is the service provider's agent, not the agent of the consumer, consumers are at risk of exploitation when they use the assistant to make important decisions.

As suggested by propositions 2 and 5, social interaction with AIVAs opens another domain that may require consumer protection. As in many other forms of two-way digital interactions, consumer "inputs" to AIVAs are expected to be coded as data for use by parent firms. This audio data is likely already being used in aggregate to train and/or refine decision algorithms, just as Google uses queries to refine their search engine. However, voice can reveal significant personal identifying information. If used to develop the algorithms' personalization or individual-level targeting, it could drive inequality and discrimination via differences in the information provided across consumer segments.

Vocal speech includes linguistic information (syntax, semantics, pragmatics), prosody, personal noises (coughs), and other auditory information (pitch, tone, volume, and rate), which can reveal demographic traits about the speaker such as gender (Schuller et al. 2013). Race and/or ethnicity can be decoded from dialects, accents, and pragmatics. Word choice can also signal social or economic strata and the use of specific slang or jargon offers further potential to refine consumer-relevant subgroup membership. Emotional signals could be used by firms to determine which products to offer during vulnerable moments. ${ }^{3}$ Finally, voice data offer clues to age- or even healthrelated states which can reveal decision-relevant vulnerabilities (Giddens et al. 2013).

These voice-inferred demographics may create opportunities for exploitation. Active use of voice data, as in "dark patterns," creates decision contexts based on the firm's preferred outcomes in ways that can act against the individual's best interests (Mathur et al. 2019). These concerns reflect the tension between personalization and privacy common in many types of digital services. In addition, the ability to do this type of identification may not be obvious or known to the consumer. Since discrimination may occur via omission - absent options or missing information - it is more challenging for affected individuals to identify that it is taking place, and for regulatory agencies to monitor it. Overall, there is a need for policy and/or legal frameworks to address how voice interaction data is captured and used in training AIVAs. These frameworks should address fairness (equity), privacy, data collection, and transparency.

\section{Conclusion}

In this paper, we have provided an initial exploration of how consumer decisionmaking may change in the presence of AIVAs and developed six propositions as a basis for future academic research. Based on these propositions, we have also suggested specific points of attention for marketing managers and policymakers who wish to respond to the proposed changes (see Table 1). In doing so, we have highlighted that interacting with an AIVA, as compared with using traditional online purchase

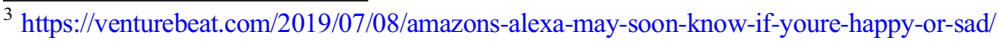


environments, presents a unique consumer experience, with implications for the decision to adopt AIVAs and for the decision process itself. We hope these topics offer a useful framing for further thinking and research in this rapidly developing and high impact area of consumer-firm interaction.

Open Access This article is licensed under a Creative Commons Attribution 4.0 International License, which permits use, sharing, adaptation, distribution and reproduction in any medium or format, as long as you give appropriate credit to the original author(s) and the source, provide a link to the Creative Commons licence, and indicate if changes were made. The images or other third party material in this article are included in the article's Creative Commons licence, unless indicated otherwise in a credit line to the material. If material is not included in the article's Creative Commons licence and your intended use is not permitted by statutory regulation or exceeds the permitted use, you will need to obtain permission directly from the copyright holder. To view a copy of this licence, visit http://creativecommons.org/licenses/by/4.0/.

\section{References}

Alba, J. W., \& Hutchinson, J. W. (1987). Dimensions of consumer expertise. Journal of Consumer Research, 13(4), 411-454.

Arentze, T. A., Dellaert, B. G. C., \& Chorus, C. G. (2015). Incorporating mental representations in discrete choice models of travel behavior: Modeling approach and empirical application. Transportation Science, 49(3), 577-590.

Baker, T., \& Dellaert, B. (2018). Regulating Robo advice across the financial services industry. Iowa Law Review, 103, 713-750.

Castelo, N., Bos, M. W., \& Lehmann, D. R. (2019a). Task-dependent algorithm aversion. Journal of Marketing Research, 56(5), 809-825.

Castelo, N., Schmitt, B., \& Sarvary, M. (2019b). Human or robot? Consumer responses to radical cognitive enhancement products. Journal of the Association for Consumer Research, 4(3), 217-230.

Diehl, K. (2005). When two rights make a wrong: Searching too much in ordered environments. Journal of Marketing Research, 42(3), 313-322.

Diehl, K., Kornish, L. J., \& Lynch, J. G. (2003). Smart agents: When lower search costs for quality information increase price sensitivity. Journal of Consumer Research, 30(1), 56-71.

Epley, N., Schroeder, J., \& Waytz, A. (2013). Motivated mind perception: Treating pets as people and people as animals. In S. Gervais (Ed.), Nebraska symposium on motivation (Vol. 60, pp. 127-152). Springer: New York.

Fast, N. J., \& Schroeder, J. (2020). Power and decision making: New directions for research in the age of artificial intelligence. Current Opinion in Psychology, 33, 172-176.

Ge, X., Brigden, N., \& Häubl, G. (2015). The preference-signaling effect of search. Journal of Consumer Psychology, 25(2), 245-256.

Giddens, C. L., Barron, K. W., Byrd-Craven, J., Clark, K. F., \& Scott Winter, A. (2013). Vocal indices of stress: A review. Journal of Voice, 27, 390-e21.

Griffin, J. G., \& Broniarczyk, S. M. (2010). The slippery slope: The impact of feature alignability on search and satisfaction. Journal of Marketing Research, 47(2), 323-334.

Harvey, N., \& Fischer, I. (1997). Taking advice: Accepting help, improving judgment, and sharing responsibility. Organizational Behavior and Human Decision Processes, 70(2), 117-133.

Häubl, G. and Trifts, V. (2000). Consumer decision making in online shopping environments: The effects of interactive decision aids. Marketing Science, 19(1), 4-21.

Häubl, G., Dellaert, B. G. C., \& Donkers, B. (2010). Tunnel vision: Local behavioral influences on consumer decisions in product search. Marketing Science, 29(3), 438-455.

Kim, S. Y., Schmitt, B. H., \& Thalmann, N. M. (2019). Eliza in the uncanny valley: Anthropomorphizing consumer robots increases their perceived warmth but decreases liking. Marketing Letters, 30(1), 1-12.

Lieberman, A., \& Schroeder, J. (2020). Two social lives: How differences between online and offline interaction influence social outcomes. Current Opinion in Psychology, 31, 16-21.

Loewenstein, G., Cain, D. M., \& Sah, S. (2011). The limits of transparency: Pitfalls and potential of disclosing conflicts of interest. American Economic Review, 101(3), 423-428. 
Lucas, G. M., Gratch, J., King, A., \& Morency, L. P. (2014). It's only a computer: Virtual humans increase willingness to disclose. Computers in Human Behavior, 37, 94-100.

Mathur, A., Acar, G., Friedman, M. J., Lucherini, E., Mayer, J., Chetty, M., \& Narayanan, A. (2019). Dark patterns at scale: Findings from a crawl of $11 \mathrm{~K}$ shopping websites. In Proceedings of the ACM on Human-Computer Interaction (Vol. 3, pp. 1-32). CSCW.

Meißner, M., Oppewal, H., \& Huber, J. (2020). Surprising adaptivity to set size changes in multi-attribute repeated choice tasks. Journal of Business Research, 111, 163-175.

Mori, M., MacDorman, K. F., \& Kageki, N. (2012). The uncanny valley: The original essay by Masahiro Mori. IEEE Spectrum, 98-100.

Munz, K, \& V Morwitz (2019). Not-so easy listening: Roots and repercussions of auditory choice difficulty in voice commerce. Available at SSRN: https://ssrn.com/abstract=3462714 or https://doi.org/10.2139 /ssrn.3462714

Nedungadi, P. (1990). Recall and consumer consideration sets: Influencing choice without altering brand evaluations. Journal of Consumer Research, 17(3), 263-276.

Newman, D. T., Fast, N. J., \& Harmon, D. J. (2020). When eliminating bias isn't fair: Algorithmic reductionism and procedural justice in human resource decisions. Organizational Behavior and Human Decision Processes, 160, 149-167.

Payne, J. W., Bettman, J. R., \& Johnson, E. J. (1993). The adaptive decision maker. Cambridge, UK: Cambridge University Press.

Raveendhran, R., \& Fast, N. J. (2019a). Technology and social evaluation: Implications for individuals and organizations. In R. N. Landers (Ed.), The Cambridge Handbook of Technology and Employee Behavior. Cambridge, UK: Cambridge University Press.

Raveendhran, R., \& Fast, N. J. (2019b). Humans judge, algorithms nudge: When and why people embrace behavior tracking. Working manuscript, University of Southern California.

Ryan, R. M., \& Deci, E. L. (2000). Self-determination theory and the facilitation of intrinsic motivation, social development, and well-being. American Psychologist, 55(1), 68.

Sawchak, M. W., \& Shelton, T. D. (2017). Exposing the fault lines under state UDAP statutes. Antitrust Journal, 81, 903-909.

Schroeder, J., \& Epley, N. (2015). The sound of intellect: Speech reveals a thoughtful mind, increasing a job candidate's appeal. Psychological Science, 26, 877-891.

Schroeder, J., \& Epley, N. (2016). Mistaking minds and machines: How speech affects dehumanization and anthropomorphism. Journal of Experimental Psychology: General, 145, 1427-1437.

Schuller, B., Steidl, S., Batliner, A., Burkhardt, F., Devillers, L., MüLler, C., \& Narayanan, S. (2013). Paralinguistics in speech and language-State-of-the-art and the challenge. Computer Speech \& Language, 27(1), 4-39.

Shu, S. B. (2008). Future-biased search: The quest for the ideal. Journal of Behavioral Decision Making, 21(4), 352-377.

Simon, D., \& Spiller, S. A. (2016). The elasticity of preferences. Psychological Science, 27(12), 1588-1599.

Slovic, P. (1972). From Shakespeare to Simon: Speculations - And some evidence - About man's ability to process information. Oregon Research Institute Research Bulletin, 12(2).

Soman, D., \& Shi, M. (2003). Virtual progress: The effect of path characteristics on perceptions of progress and choice. Management Science, 49(9), 1229-1250.

Steffel, M., \& Williams, E. F. (2018). Delegating decisions: Recruiting others to make difficult choices. Journal of Consumer Research, 44(5), 1015-1032.

Steffel, M., Williams, E. F., \& Perrmann-Graham, J. (2016). Passing the Buck: Delegating choices to others to avoid responsibility and blame. Organizational Behavior and Human Decision Processes, 135, 32-44.

Usta, M., \& Haubl, G. (2011). Self-regulatory strength and consumers' relinquishment of decision control: When less effortful decisions are more resource depleting. Journal of Consumer Research, 48(2), 403-412.

Wang, S., Lilienfeld, S. O., \& Rochat, P. (2015). The uncanny valley: Existence and explanations. Review of General Psychology, 19(4), 393-407.

West, P. M., Brown, C. L., \& Hoch, S. J. (1996). Consumption vocabulary and preference formation. Journal of Consumer Research, 23(2), 120-135.

Xiao, B., \& Benbasat, I. (2007). E-commerce product recommendation agents: Use, characteristics, and impact. MIS Quarterly, 31(1), 137-209.

Yalcin, Ö. N., \& DiPaola, S. (2018). A computational model of empathy for interactive agents. Biologically Inspired Cognitive Architectures, 26, 20-25.

Publisher's note Springer Nature remains neutral with regard to jurisdictional claims in published maps and institutional affiliations. 


\section{Affiliations}

Benedict G. C. Dellaert ${ }^{1,2} \cdot$ Suzanne B. Shu ${ }^{3}$. Theo A. Arentze ${ }^{4} \cdot$ Tom Baker $^{5}$. Kristin Diehl ${ }^{6} \cdot$ Bas Donkers $^{1} \cdot$ Nathanael J. Fast ${ }^{6} \cdot$ Gerald Häubl $^{7} \cdot$ Heidi Johnson $^{8}$ • Uma R. Karmarkar ${ }^{9}$ • Harmen Oppewal ${ }^{2}$ • Bernd H. Schmitt ${ }^{10}$ • Juliana Schroeder $^{11} \cdot$ Stephen A. Spiller ${ }^{12}$ - Mary Steffel $^{13}$

Suzanne B. Shu

suzanne.shu@cornell.edu

Theo A. Arentze

T.A.Arentze@tue.nl

Tom Baker

tombaker@law.upenn.edu

Kristin Diehl

kdiehl@marshall.usc.edu

Bas Donkers

donkers@ese.eur.nl

Nathanael J. Fast

nathanael.fast@marshall.usc.edu

Gerald Häubl

gerald.haeubl@ualberta.ca

Heidi Johnson

hjohnson@ finhealthnetwork.org

Uma R. Karmarkar

ukarmarkar@ucsd.edu

Harmen Oppewal

harmen.oppewal@monash.edu

Bernd H. Schmitt

bhs1@gsb.columbia.edu

Juliana Schroeder

jschroeder@berkeley.edu

Stephen A. Spiller

Stephen.spiller@anderson.ucla.edu

Mary Steffel

m.steffel@neu.edu

Erasmus School of Economics, Erasmus University, Rotterdam, Netherlands

2 Monash Business School, Monash University, Caulfield East, Australia

3 Dyson School of Applied Economics and Management, Cornell University, Ithaca, NY, USA

4 Department of the Built Environment, Eindhoven University of Technology, Eindhoven, Netherlands

5 University of Pennsylvania Law School, University of Pennsylvania, Philadelphia, PA, USA

6 Marshall School of Business, University of Southern California, Los Angeles, CA, USA 
7 Alberta School of Business, University of Alberta, Edmonton, Canada

$8 \quad$ Financial Health Network, Washington, D.C., USA

9 Rady School of Management and School of Global Policy and Strategy, UC San Diego, San Diego, CA, USA

10 Columbia Business School, Columbia University, New York, NY, USA

11 Haas School of Business, University of California at Berkeley, Berkeley, CA, USA

12 Anderson School of Management, UCLA, Los Angeles, CA, USA

13 D'Amore-McKim School of Business, Northeastern University, Boston, MA, USA 